\title{
Home Compatible Omnidirectional Hovercraft Robot
}

\author{
Gergely Nagymáté \\ Dept. of Mechatronics Optics and Engineering Informatics \\ Budapest University of Technology and Economics \\ Budapest, Hungary \\ nagymate.gergely@gmail.com
}

\author{
Zita V. Farkas \\ Dept. of Machine and Product Design \\ Budapest University of Technology and Economics \\ Budapest, Hungary \\ zita.farkas@mail.bme.hu
}

\begin{abstract}
As robots slowly integrate into home environments, synthesis of navigation, maneuverability and human acceptance is inevitable. This paper introduces a holonomic hovercraft design and the associated omnidirectional controlling algorithm. Hovercraft capabilities were investigated and discussed though design recommendations in relation to a robot compatible environment. The main aim of the design was to achieve better maneuverability, enhanced capabilities of overcoming obstacles, and the elimination of the drift phenomena that is a characteristic of conventional underactuated hovercraft designs, where rear rotor drive exerts thrust in one direction. Due to own inertia and the low friction of the air cushion, the hovercraft slips out in the original direction. Beyond solving this drift problem, another key feature of our design is the capability to be controlled in a global reference frame regardless of its orientation and desired trajectory with the help of a holonomic thruster drive. Orientation control is also implemented by turning the base of the thrusters. The design was implemented on a remote controlled hovercraft robot and proved to have a superior maneuverability over conventional hovercraft designs, thus our research greatly contributes to future human-robot cooperation in the living environment.
\end{abstract}

Keywords-hovercraft robot; omnidirectional drive; orientation control; robot compatible environment

\section{INTRODUCTION}

Rapid development of service robots [1] is going to emerge in everyday environments, where synthesis of navigation, maneuverability and human acceptance is managed under various conditions, in order to successfully execute given tasks. Taking the robot's capabilities into account and synchronizing them with the built environment, the robot compatible environment is created [2], where mobile robots can navigate and maneuver with high efficiency. Operational reliability and safety are the basis for hovercraft robots as well, to integrate them efficiently into the environment, that is cluttered with obstacles. Where detecting information, reaching for objects, maneuverability, user friendly communication are basic necessities [3], harmonization of built environment in alignment with digital, physical and intelligent space becomes emphasized [4][5]. This research investigates spatial characteristics of surrounding environment in relation to hovercraft robots and gives recommendations to create an environment that enhances navigation parallel to risk reduction and hazard prevention [6][7]. In the near future the robot will be used by non engineers or not robot specialist [8]. In the aspect of robot users people can be divided to 4 main groups[9].

\section{- robot specialist engineer \\ - engineer, but not robot specialist \\ - not engineer, but being interested in robotics \\ - elderly people, reluctant to robotics}

For robots in our daily life it is not enough to execute a preprogrammed action line. They must be able to adopt themselves to changing environment, make their own decisions and in addition, they have to socially fit into the human environment. In the discussion design recommendations for hovercraft robot environment compatibility are introduced.

This paper presents a unique hovercraft design and control method designed for agile maneuverability in narrow places between the target boxes. The robot is also controlled in the global coordinate system of the human operator, unlike conventional hovercraft, where the trajectory and maneuvers depend on the robot orientation. Conventional hovercraft robots are underactuated and also suffer from "drift". They have rear rotor drive that can exert thrust in one direction. During cornering, due to the own inertia of the hovercraft and the low friction of the air cushion, the hovercraft will slip out in the original direction. There are control methods that improve controllability of these hovercrafts for example with switching Fuzzy controller [10]. Other methods aim to minimize sway velocity error and heading error [11].

The assigned task is easier to perform with a holonomic hovercraft. An omnidirectional design should be able to eliminate drift and can be controlled in a global reference frame regardless of its orientation and desired trajectory. An autonomous hovercraft robot presented by Roubieu and Serres is based on a conventional hovercraft design and uses two additional lateral thrusters for sideways movements [12]. Orientation change is achieved with the differential drive of the two rear thrusters. This solution does not fulfill our requirements. A robot presented by Detweiler, Griffin and Roehr implements omnidirectional drive with three thrusters positioned in 120 degrees generating thrust in arbitrary 
directions [13]. They use two additional thrusters for rotating the robot. This solution separates the omnidirectional drive from the orientation control, however requires extra thrusters. If they are used for orientation regulation, then they should usually oscillate around zero revolution. This is a costly solution and less effective.Our design is also carried out with three thrusters positioned in 120 degrees to preserve the benefits of omnidirectional driving. On the other hand, the orientation regulation is implemented by turning the bases of the thrusters. This solution spares two thrusters, but requires a servo motor and synchronous turning of the bases.

The paper is distributed as follows: Section II describes the design and the parts of the robot; Section III contains a discussion on the implemented orientation regulation; Section IV introduces the omnidirectional control algorithm; Section V discusses application in robot compatible environment; and finally, conclusions are drawn in Section VI.

\section{PARTS AND DESIGN}

The base of the robot is an octagonal carbon fiber composite rigid chassis. This is the base of the omnidirectional drives. The air cushion of the hovercraft is produced by a lifting propeller and a flexible plastic skirt that hangs down from the chassis. The thrusters can be rotated simultaneously by a hidden toothed belt under the chassis driven by an RC servo motor. The thrusters are iPower 2208/14 type brushless DC (BLDC) motors with propellers. The motor consoles and propeller safety grids just like many other fitting on the robot are unique, 3D printed parts. In the middle of the chassis there is the lifting motor, which is the same type BLDC with double propellers for higher air transportation. Above the lifting motor there are the LiPo battery and the electronics on a carbon pipe console, which leaves enough space for the air inlet of the lifting motor. The electronics is based on a STM32F3 Discovery board that carries a STM32F303VCT6 ARM Cortex-M4 MCU, LSM303DLHC 3 axis accelerometermagnetometer IC and a L3GD20 digital output gyroscope. A 3 DoF robotic arm is placed above the electronics that can carry and place the dices and is capable of removing dices of opponent robots. The arm is actuated by high power RC servos and closed during robot navigation to achieve a centralized mass distribution, keeping the air cushion of the hovercraft homogenous. The design is shown in Fig. 1.

Omnidirectional driving requires similar characteristic for the three thrusters which are desirably linear. BLDC motors are paired with electronic speed controllers (ESC) and propellers and are characterized as one unit. The input value is the PWM duty cycle of the ESCs, whereas the output value is the thrust generated by the motor-propeller combinations. The motors were attached to a console such way that they exert thrust downwards. This installation was placed on a digital scale to measure thrust generated by different duty cycles.

Fig. 2 shows the result of the measurements. It can be seen that the characteristics are not strictly linear but linear enough for our controller. However, one of the three motors shows a slightly more flat characteristic that was easily compensated with a constant multiplication.

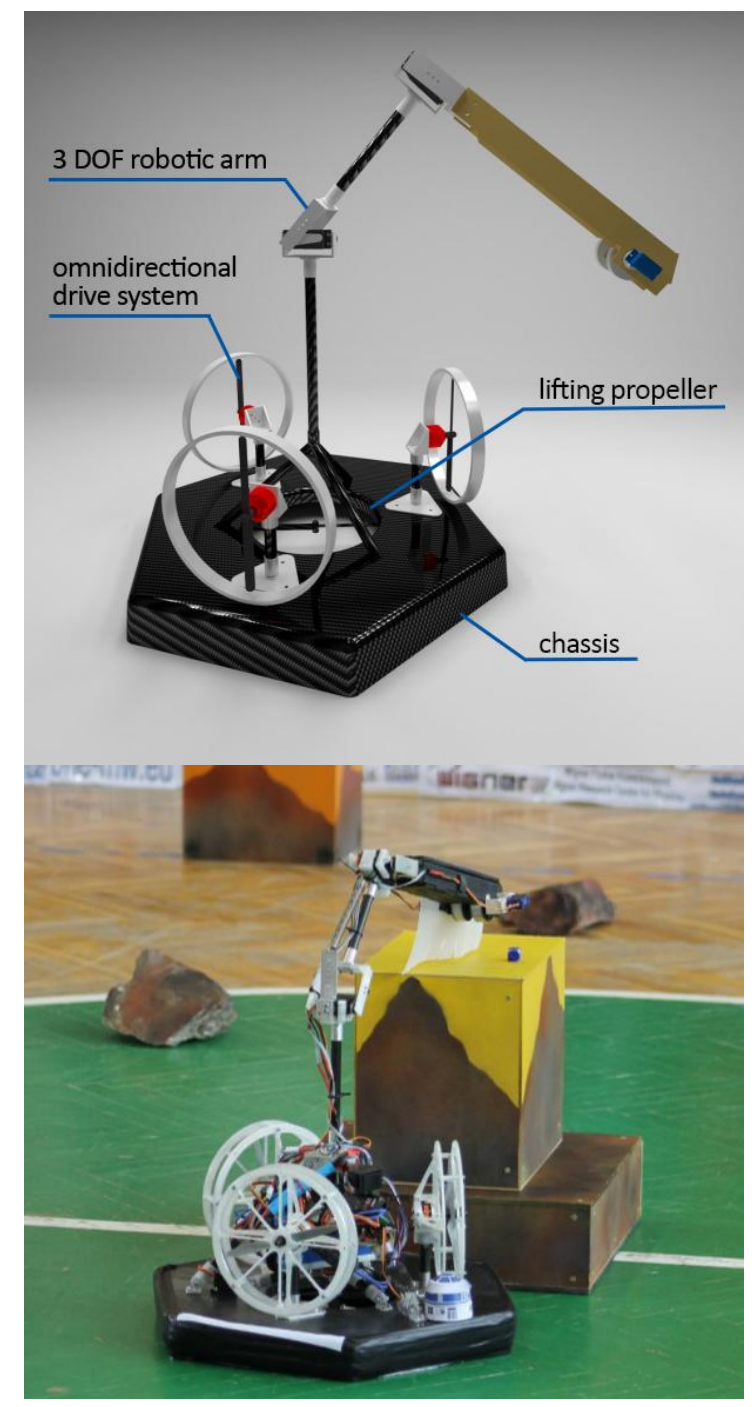

Fig. 1. Design plan and the manufactured robot in operation

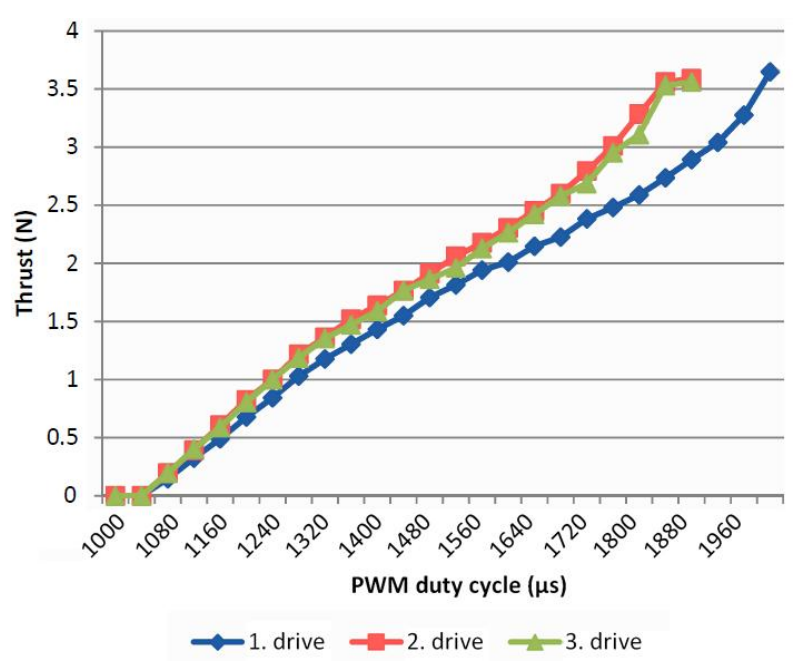

Fig. 2. Thruster characteristics 


\section{MAINTAINING ORIENTATION}

Perpetual knowledge of vehicle orientation is essential in the global frame controlling. The robot is equipped with inertial measurement unit and compass sensor, thus a complete Kalman based algorithm or a simpler gradient descent orientation estimation algorithm could be used to calculate proper three dimensional orientations [14]. This vehicle moves in plane with three degrees of freedom tied. The only possible rotation is the heading rotation around the vertical axis. Pitch and roll angles naturally never change, therefore there is no use of the accelerometer readings in the sensor fusion. The compass sensor could have served a great role in measuring absolute orientation. However, the magnetometer sensor proved to be useless on this small robot due to the expansive magnetic field of the BLDC motors. The simple final solution uses only one data from the gyroscope. It integrates the angular velocity around the vertical axis compensated with the offset output of the gyroscope. This offset is measured every time the hovercraft is settled. This simple solution proved to be surprisingly reliable for several minutes of operation. Since the hovercraft is remote controlled by a human, it is the role of the operator to send $\pm 5^{\circ}$ compensation instructions to the robot controller, to compensate long term disorientation.

The orientation is finally maintained with a simple PID controller tuned with the Ziegler- Nichols method [15]. The output of the controller is the torque that compensates the constant torque of the lifting propeller and keeps the robot in the desired orientation, which is basically the initial orientation.

\section{OMNIDIRECTIONAL CONTROL ALGORITHM}

The control algorithm is separated to calculation of basic forces, their rotation and compensation to yield desired torque.

\section{A. Calculating Basic Forces for Omnidirectional Drive}

The $\mathrm{x}-\mathrm{y}$ reference forces are given by the operator as desired direction and acceleration in the global coordinate system. In certain cases the robot orientation is changed by the operator. Therefore, the reference forces are transformed into the hovercraft coordinate system with the following equations:

$$
\begin{aligned}
& F_{x}=F_{x_{\text {ref }}} \cdot \cos (-\gamma)-F_{y_{\text {ref }}} \cdot \sin (-\gamma) \\
& F_{y}=F_{y_{\text {ref }}} \cdot \cos (-\gamma)+F_{x_{\text {ref }}} \cdot \sin (-\gamma)
\end{aligned}
$$

where $F_{x}$ and $F_{y}$ are the desired forces in the robot coordinate system, $F_{x r e f}$ and $F_{y r e f}$ are in the global coordinate system and $\gamma$ is the robot orientation angle relative to the global y axis. Every further calculation is also interpreted in the robot reference frame.

The $x-y$ forces then transformed to polar coordinates with equation 3 and 4 .

$$
\begin{aligned}
\beta & =\operatorname{arctg} \frac{F_{y}}{F_{x}} \\
F_{a b s} & =\sqrt{F_{x}^{2}+F_{y}^{2}}
\end{aligned}
$$

Relation between robot coordinate system and world coordinate system is shown in Fig. 3.

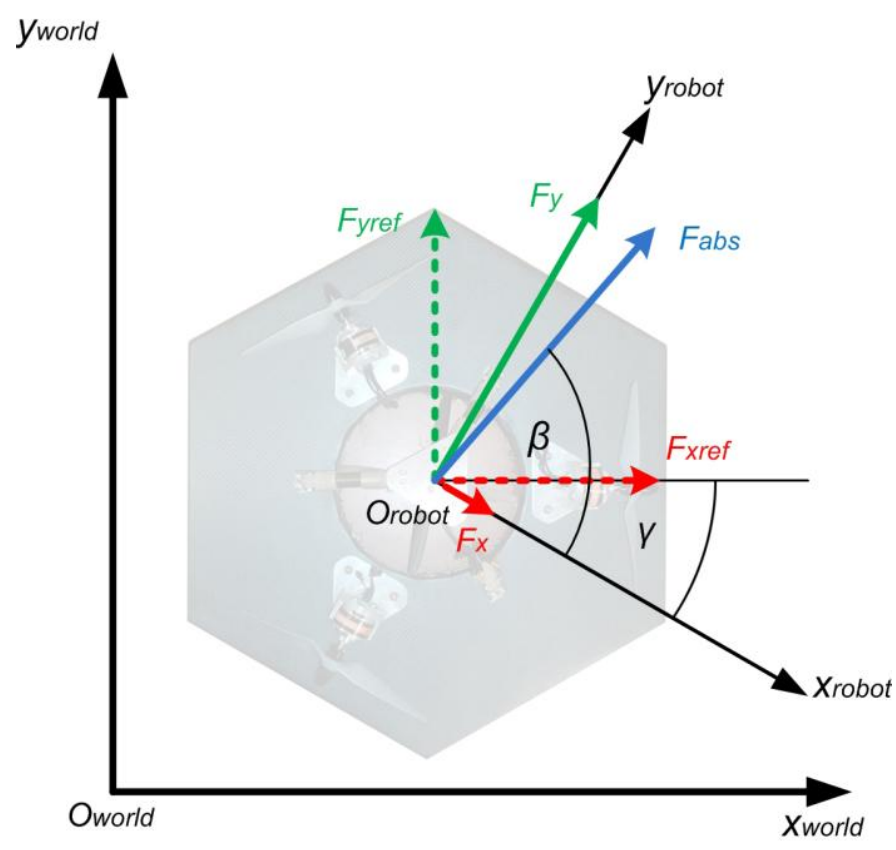

Fig. 3. Robot coordinate system in relation to world coordinate system

The lines of action of the thrusts intersect in the robot's centerpoint with enclosed angles of 120 degrees. The propellers exert thrust only in one direction. $F_{a b s}$ could be basically decomposed onto the two adjacent lines of action. However, there will always be a minimal thrust exerted on the third line of action. This is due to the consideration that none of the motors is shot down completely any of the motors during operation. BLDC motors take some time to run up correctly. With an input signal taking them to a minimal feasible speed, a smoother control can be achieved. This minimum speed exerts $6 \%$ of the maximum thrust that works against the two effective forces. The $\mathrm{C}$ code snippet that is used to decompose $F_{a b s}$ to the basic forces taking the minimal reverse thrust into account is shown below.

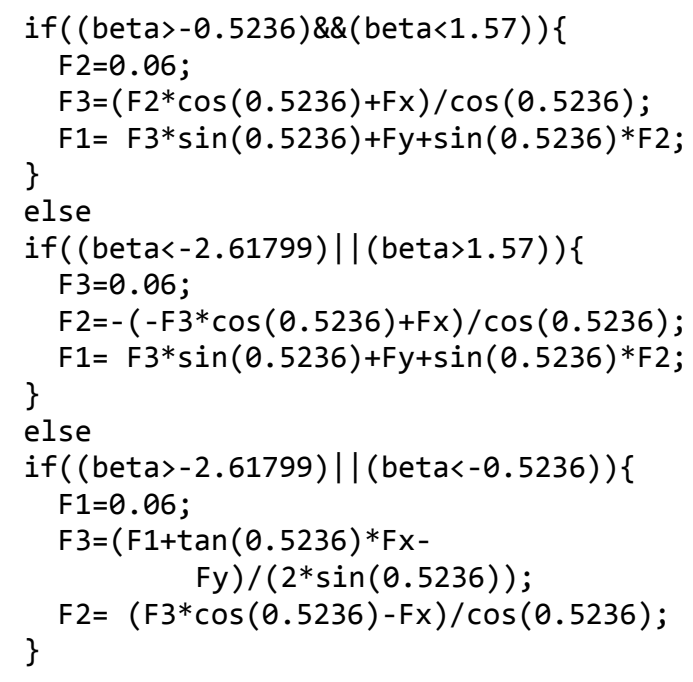

Result of the decomposition is shown in Fig. 4, in two example pictures taken from a LabVIEW simulation. 


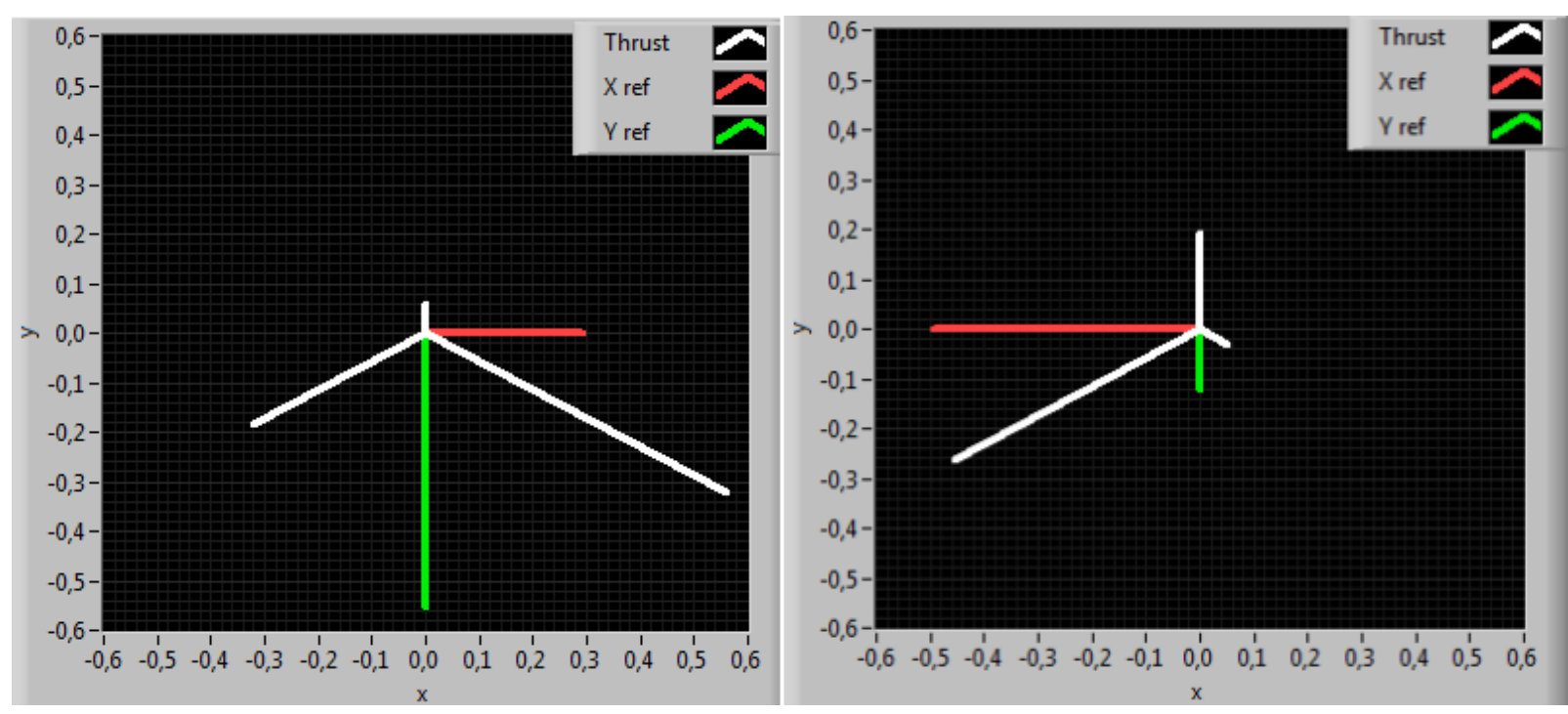

Fig. 4. $F_{x}$ and $F_{y}$ reference forces in the robot coordinate system are decomposed to the three lines of action of the driving motors

\section{B. Compensate Thrust with Required Torque}

The orientation regulator calculates the required torque to keep robot orientation steady. This torque is obtained by turning the lines of action of thrust generating motors, so that they do not cross the robot's center of mass. Force components $\left(F_{r 1}, F_{r 2}, F_{r 3}\right)$ appear perpendicular to the basic forces defined by the omnidirectional drive $\left(F_{a 1}, F_{a 2}, F_{a 3}\right)$. Fig. 5 shows the described forces, where $M$ is the desired torque, $\alpha$ is the rotated angle of the motors in the algorithm, $F_{m 1}, F_{m 2}, F_{m 3}$ are the forces finally applied by the motors and $r$ is the distance of the motors' rotary bases from the center.

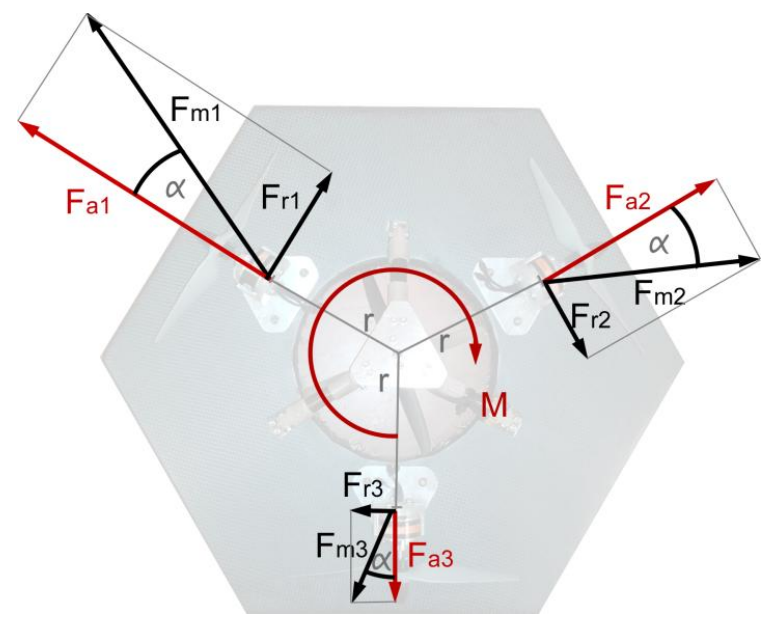

Fig. 5. Force vectors and required torque

The required torque is the sum of the applied torques by tangential forces:

$$
M=F_{r 1} \cdot r+F_{r 2} \cdot r+F_{r 3} \cdot r
$$

These forces can be expressed with the rotation angle and the known radial forces required for the omnidirectional drive:

$$
M=F_{a 1} \cdot \tan \propto \cdot r+F_{a 2} \cdot \tan \propto \cdot r
$$

$$
+F_{a 3} \cdot \tan \propto \cdot r
$$

Rotation angle can be expressed from the above equation:

$$
\propto=\operatorname{arctg}\left(\frac{M}{\left(F_{a 1}+F_{a 2}+F_{a 3}\right) \cdot r}\right)
$$

Knowing the rotation, applied forces by the motors can be calculated as follows:

$$
\begin{aligned}
& F_{m 1}=\frac{F_{a 1}}{\cos \alpha} \\
& F_{m 2}=\frac{F_{a 2}}{\cos \alpha} \\
& F_{m 3}=\frac{F_{a 3}}{\cos \propto}
\end{aligned}
$$

Prior these calculations a conditional addition is applied. If the sum of the basic forces does not reach a specified minimum then balanced forces are added to them, so $\alpha$ rotations larger than 40 degrees will not be required. This is necessary due to mechanical limitations and to reach smoother orientation control. The mentioned minimum force depends on the applied required torque.

\section{DISCUSSION}

The proposed hovercraft is a suitable application in the robot compatible environment. Hovercraft robots compared to wheeled robots stand out in their capability to slide over the air cushion. Steering in case of conventional hovercraft robots is different from holonomic hovercraft or wheeled drive, and direct drives used as mobile robot platforms. In case of conventional hovercraft steering motor is coupled with a thruster so as to thrust air along the axis of thruster supported by rudder blades (perpendicular to the axis of thruster). As rudder thrust air is deflected to left or right, steering the robot laterally, drifting is a negative aspect. In case of a holonomic hovercraft drift problem is overcome by using 3 thrusters positioned in 120 degrees work together without the need of rudder blades, thus sufficient maneuverability is achieved. 
Hovercraft can move over uneven surface and initial traction is not needed compared to wheeled robots. Still hazardous maneuvers can be problematic, thus in case of movement over a ramp, low steepness is necessary for the robot to avoid instability or losing track. Longer ramps need proper edge protection, meanwhile should not be obtrusive for human user. Ramp design requires extra space before and after the ramp, it shall be designed to leave turning diameter and motor torque to roll onto and off the ramp safely.Floor surface with openings in case of wheeled robots leads to stuck wheels, while in the case of hovercraft,air cushion leakage that leads to movement stop. When a carpet has too high piles or a surface is too bumpy it can also lead to stop or hazardous maneuvering. It is important to examine the robot and its environment for damage concerns regarding impact resistance, entrapment avoidance and height differences.

To achieve navigational tasks, knowledge of robot dimensions is necessary: width, length, height, turning diameter, situation of manipulator arms, weight, possible speed and motor torque. Turning space and maneuvering space should be adequate. $T D_{H}$ turning diameter of a holonomic robot is the minimum turning diameter, it is the same as robot's overall diameter and turning midpoint resides at the center point of the robot, where $X$ is the maximum width and $Y$ is the maximum length of the robot:

$$
T D_{H}=\sqrt{X^{2}+Y^{2}}
$$

$T D_{D I R}$ turning diameter of a direct drive robot is the minimum turning diameter, which is approximately twice the size of the maximum diameter of the robot. Where $X$ is the width and $Y$ is the length of the robot, $D$ is the distance between the inner fixed and front steered wheel and $\delta$ is the maximum steering angle:

$$
T D_{D I R}=2 \times \sqrt{\left(X+\frac{D}{\tan \delta}\right)^{2}+Y^{2}}
$$
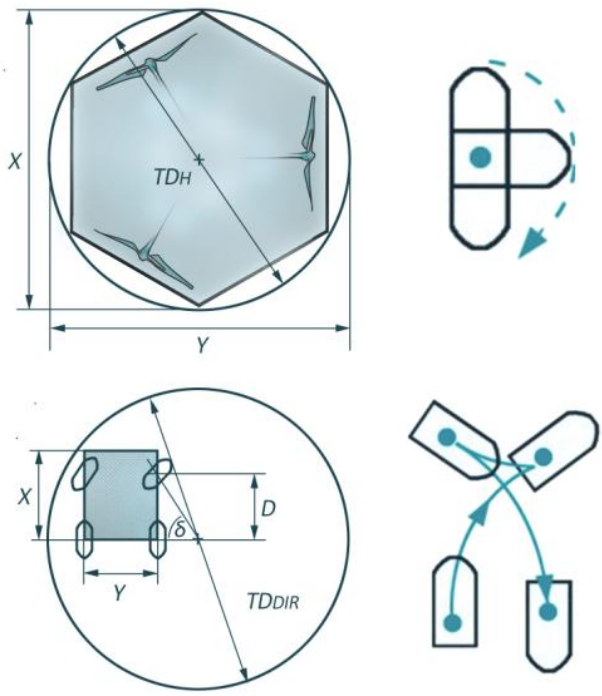

Fig. 6. Turning diameter and maneuvering of holonomic and direct drives
Maneuvering requires turn in reverse direction in a narrow space in case of direct drive, as shown in Fig. 6. While omnidirectional movement in 3 DoF enables not only sensors or actuators, but the whole body of the robot to have different orientation from the actual moving direction.

Turning with a holonomic hovercraft robot is achieved by simultaneous thrust vectoring and anti-torque control. There is pressurized air cushion underneath the chassis, when loaded it needs to be balanced centrally, not to let air escape from under the skirt. Stopping of the robot is created with calibrated thrusters, and decreasing lift propeller speed, thus holonomic hovercraft can stop efficiently.

In an indoor environment problems of hovercraft robots are noise and air disturbance. There is the noise of the motors but noise level originating from thrusters is quite high, factors like diameter of blades and blade tip form are taking part in noise generation. With bigger diameter of blades greater force can be achieved with slower propeller speed, thus less vibration and noise. With the evolution of propellers, unique thruster blade surface and edge design with enclosed tunnel like casing will be possible, that creates less turbulence, quiet propelling and greater ratio of directed air, which can lead to more precise maneuvering. In the future, when robot and human share living space in the home, sound levels are an important consideration, quieter airflow will meet a better acceptance. Motors encapsulated within robot case can lower noise level. Still, in an environment, where indoor or outdoor area covers great distances and noise is not a factor hovercraft robots are already advantageous.

While most robots are restricted indoors, hovercraft robots are able to explore outdoor environments with uneven surface characteristics. In many institutions, like hospitals, communal homes, multi-building parks, from lengthy corridors to in between buildings areas, they can be used for transporter or courier purposes (even with human guidance), that would be difficult or significantly slower for a wheeled robot. Moreover hovercraft needs fewer resources for the same distance, due to its speed and weight, energy usage is lower, that translates in lower $\mathrm{CO}_{2}$ emission.

To cross height gaps or prevent tripping, adequate gradual ramps are needed for robots. Many ramps have been built due to accessible built environment regulations. There is no need for further construction works or disturbance of the built facilities.

While wheeled robots that are prone to get stuck in between chair legs, a holonomic robot can manage to continue its movement by dynamically adjusting thruster angles when approaching an obstacle. Holonomic hovercraft robot can change its orientation during movement, thus more natural and flexible driving is possible. In an environment where furniture or obstacles are present ( $2 \mathrm{~cm}$ doorsills, chair legs, carpets, cords or cable covers on the ground) robot still needs to be capable of seamless maneuvers as shown in Fig. 7 and Fig. 8. 

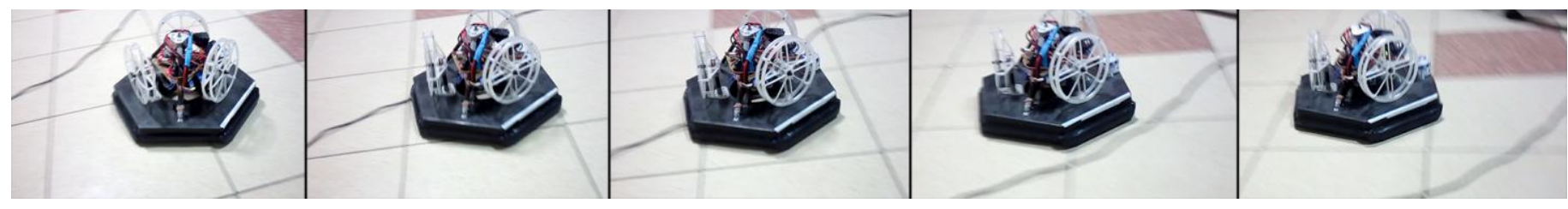

Fig. 7. Holonomic hovercraft robot crossing over cord

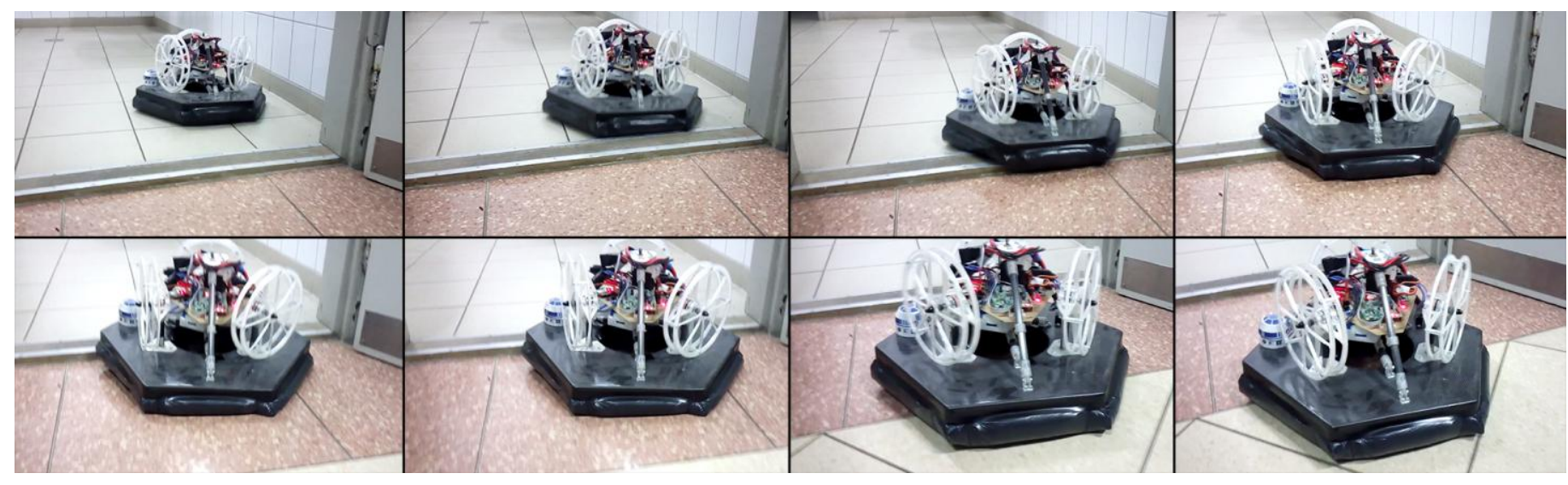

Fig. 8. Holonomic hovercraft robot crossing over a $2 \mathrm{~cm}$ high doorsill

In regard to the skirt design of the hovercraft's skirt less turbulent, smooth airflow is created with circular shape restricting the outward expansion of skirt. Outer surface of skirt needs enhanced durability and semi-rigid structure that reduces friction and wear out during crossing over obstacles or approaching/leaving ramps, while keeping elasticity that helps movement continuity and prevents damage of object or injury of human. Propellers or other rotating parts need safety guard, while not limiting airflow.

In regard to maintenance, wheeled robots collect dust around their wheel bearings and spinning parts, or can get stuck in carpet pile, hovercraft robot is less prone to this effect. Nevertheless, periodic audits and reviews of robot and its environment must be ensured to determine maintenance of safe and risk free operation.

\section{CONCLUSION}

The omnidirectional hovercraft design and control algorithm described in this paper opens new perspectives in design of hovercraft type robots. This design offers superior maneuverability in narrow places and also simpler controllability by automation or remote controlling. Users require service tasks from robot, thus robot needs balanced robot compatible environment to be compliant with those requirements. During the process of integrating robots into the everyday environment, that combines navigation, adaptability and accessibility, hovercraft robots greatly contribute to future use and integration of safe, reliable and effective human-robot cooperation.

\section{ACKNOWLEDGMENT}

We thank for the financial support given by Lightware Ltd., MOGI Department of Budapest Univ. of Technology and
Economics and the Mechatronical Department of Student College of Mechanical Engineering. Authors gratefully appreciate the "MaMSTAR" team of mechatronics engineering students for the common work on the robot.

\section{REFERENCES}

[1] Japanese robotics forecast, (2010) [Online]. Available: www.meti.go.jp/english/press/data/20100423_01.html

[2] Z. V. Farkas, P. Korondi, and L. Fodor, "Safety aspects and guidelines for robot compatible environment," In: 38th Annual Conference on IEEE Industrial Electronics Society (IECON 2012) pp. 5547-5552

[3] B. Mutlu, J. Forlizzi. "Robots in organizations: the role of workflow, social, and environmental factors in human-robot interaction," HRI ' 08 Proceedings of the 3rd ACM/IEEE international conference on Human robot interaction, Amsterdam, The Netherlands, March 12-15, 2008

[4] C. A. Smarr, C. B. Fausset and W. A. Rogers. "Understanding the Potential for Robot Assistance for Older Adults in the Home Environment," Technical Report HFA-TR-1102, Georgia Institute of Technology, 2011

[5] M. Niitsuma, W. Beppu, T. Ichikawa, Sz. Kovacs and P. Korondi. "Implementation of robot behaviors based on ethological approach for monitoring support system in Intelligent Space," IEEE/ASME International Conference on Advanced Intelligent Mechatronics, Budapest, Hungary, July 3-7, 2011, pp. 536-541

[6] TC 184 Automation systems and integration, SC 2 Robots and robotic devices, (2014) [Online]. Available: http://www.iso.org/iso/ iso_catalogue/catalogue_tc/catalogue_tc_browse.htm?commid= 54138

[7] Accessibility standards. (2014) [Online]. Available: http://www.humanics-es.com/recc-disabled.htm\#standards

[8] Tajti Ferenc, Korondi Péter, Kovács Bence, Szayer Géza, Dániel Balázs,"CRM TC covering paper - Robotics trends" Proceedings of the IECON 2013: IEEE, 2013. pp. 48-53.

[9] Bjorn Solvang, Gábor Sziebig, Péter Korondi,"Shop_Floor Architecture for Effective Human-Machine and Inter-Machine Interaction” ,ACTA POLYTECHNICA HUNGARICA 9:(1) pp. 183-201. (2012)

[10] K. Tanaka, M. Iwasaki, H. O. Wang, "Switching Control of an R/C Hovercraft: Stabilization and Smooth Switching," IEEE transactions on 
Recent Innovations in Mechatronics (RIiM) Vol. 2. (2015). No. 1-2.

DOI: $10.17667 /$ riim.2015.1-2/5.

systems, man, and cybernetics- Part B: Cybernetics, VOL. 31, NO. 6 , December 2001, pp. 853-863

[11] J. Zhao, J. Pang, "Trajectory Control of Underactuated Hovercraft," 8th World Congress on Intelligent Control and Automation July 6-9 2010, Jinan, China pp. 3904-3907

[12] F.L.Roubieu, J. Serres, N. Franceschini, F. Ruffier, S. Viollet, "A fully-autonomous hovercraft inspired by bees: Wall following and speed control in straight and tapered corridors," Robotics and Biomimetics (ROBIO), 2012 IEEE International Conference, Dec. 2012, pp. 13111318
[13] C. Detweiler, B. Griffin, H. Roehr, "Omni- directional hovercraft design as a foundation for MAV education", Intelligent Robots and Systems (IROS), 2012 IEEE/RSJ International Conference, Oct. 2012, pp 786792

[14] Madgwick, S.O.H. ; Dept. of Mech. Eng., Univ. of Bristol, Bristol, UK ; Harrison, A.J.L. ; Vaidyanathan, R., "Estimation of IMU and MARG orientation using a gradient descent algorithm," Rehabilitation Robotics (ICORR), 2011 IEEE International Conference, June 29 2011-July 1 2011, pp. 1-7

[15] J. G. Ziegler and N. B. Nichols, "Optimum setting for automatic controllers," Trans. ASME, vol. 64, 1942, pp. 759-768 